\title{
Community-based network analyses reveal emerging connectivity patterns of protein-protein interactions in murine melanoma secretome
}

\author{
Rodrigo Francisquini ${ }^{\mathrm{a}}$, Rafael Berton ${ }^{\mathrm{b}}$, Sandro Gomes Soares ${ }^{\mathrm{c}}$, Dayelle S. Pessotti ${ }^{\mathrm{b}}$, \\ Maurício F. Camacho ${ }^{b}$, Débora Andrade-Silva ${ }^{\mathrm{d}}$, Uilla Barcick ${ }^{\mathrm{b}}$, Solange M.T. Serrano ${ }^{\mathrm{d}}$, \\ Roger Chammas ${ }^{\mathrm{e}}$, Mariá C.V. Nascimento ${ }^{\mathrm{a}}$, André Zelanis ${ }^{\mathrm{b},}$ \\ ${ }^{a}$ Department of Science and Technology, Federal University of São Paulo, (ICT-UNIFESP), São José dos Campos, SP, Brazil \\ ${ }^{\mathrm{b}}$ Functional Proteomics Laboratory, Department of Science and Technology, Federal University of São Paulo, (ICT-UNIFESP), São José dos Campos, SP, Brazil \\ ${ }^{c}$ Department of Biochemistry, University of Cambridge, United Kingdom \\ ${ }^{\mathrm{d}}$ Laboratório de Toxinologia Aplicada, Center of Toxins, Immune-Response and Cell Signaling (CeTICS), Instituto Butantan, São Paulo, Brazil \\ e Instituto do Câncer do Estado de São Paulo, Faculdade de Medicina da Universidade de São Paulo, São Paulo, Brazil
}

\section{A B S T R A C T}

Protein-protein interaction networks (PPINs) are static representations of protein connections in which topological features such as subgraphs (communities) may contain proteins functionally related, revealing an additional layer of interactome complexity. We created two PPINs from the secretomes of a paired set of murine melanocytes (a normal melanocyte and its transformed phenotype). Community structures, identified by a graph clustering algorithm, resulted in the identification of subgraphs in both networks. Interestingly, the underlying structure of such communities revealed shared and exclusive proteins (core and exclusive nodes, respectively), in addition to proteins that changed their location within each community (rewired nodes). Functional enrichment analysis of core nodes revealed conserved biological functions in both networks whereas exclusive and rewired nodes in the tumoral phenotype network were enriched in cancer-related processes, including TGF $\beta$ signaling. We found a remarkable shift in the tumoral interactome, resulting in an emerging pattern which was driven by the presence of exclusive nodes and may represent functional network motifs. Our findings suggest that the rearrangement in the tumoral interactome may be correlated with the malignant transformation of melanocytes associated with substrate adhesion impediment. The interactions found in core and new/rewired nodes might potentially be targeted for therapeutic intervention in melanoma treatment.

Significance: Malignant transformation is a result of synergistic action of multiple molecular factors in which genetic alterations as well as protein expression play paramount roles. During oncogenesis, cellular crosstalk through the secretion of soluble mediators modulates the phenotype of transformed cells which ultimately enables them to successfully disrupt important signaling pathways, including those related to cell growth and proliferation. Therefore, in this work we profiled the secretomes of a paired set of normal and transformed phenotypes of a murine melanocyte. After assembling the two interactomes, clusters of functionally related proteins (network communities) were observed as well as emerging patterns of network rewiring which may represent an interactome signature of transformed cells. In summary, the significance of this study relies on the understanding of the repertoire of 'normal' and 'tumoral' secretomes and, more importantly, the set of interacting proteins (the interactome) in both of these conditions, which may reveal key components that might be potentially targeted for therapeutic intervention.

\section{Introduction}

Cellular communication strongly relies on the secretion of soluble mediators that, in general, interact with cell surface receptors and enable biological responses in a proper timescale for signaling processes to occur $[1,2]$. Therefore, the study of secreted proteins (the secretome) from normal cells and tumoral counterparts allows a comprehensive portrait of the so-called 'effectors' in many biological circuits, including those related to tumor development. Cell homeostasis is disrupted in many ways during oncogenesis; the main biological implications are derived from a wide range of factors, including somatic mutations, epigenetic modifications and post-transcriptional/translational modifications, which ultimately result in the modulation of the gene expression [3]. Collectively, these factors often result in rewiring connections of signaling networks in malignant cells. Since protein-protein interactions may eventually enable transformed cells to grow, proliferate and invade neighboring tissues, a comprehensive view of the imbalance caused by the oncogenic processes requires the understanding of the interplay of interactors in altered biological circuits. Pathway and network analysis are analytical approaches that reduce the data involving thousands of altered genes/proteins in complex biological systems to smaller datasets which, in turn, enable the detection and interpretation of specific

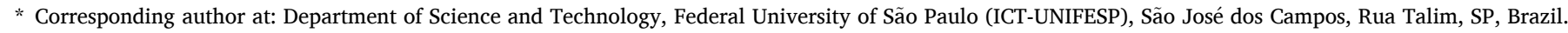

E-mail address: andre.zelanis@unifesp.br (A. Zelanis).
} 
biological questions, such as cancer-related processes, clinically distinct outcomes and drug targets [4-6]. In this respect, protein-protein interaction networks (PPINs) are static representations of protein connections in biological systems. Although this simplification lacks the dynamic nature of the real protein context, it allows the identification of specific topologies related to the patterns of connectivity of a given PPIN. Moreover, some additional network features may be observed, such as the identification of subgraphs (communities), which contain proteins that may be functionally related, revealing an additional layer of complexity among protein-protein interactions within the network. Kaushik and coworkers [7] used network analysis to study melanoma stage progression and found that melanoma-related genes can modulate their activities by rewiring network connections, independent of altered gene expression. A recent work with DNA methylation, gene expression and corresponding clinical data from breast invasive carcinoma, skin cutaneous melanoma and uterine corpus endometrial carcinoma underscored gene co-methylation networks, resulting in the identification of core gene modules which allowed the molecular typing of cancer and the prognosis of patients [8]. Such network-based approach revealed that gene modules were more reliable in cancer prognosis than gene expression profiles. Systems-level analysis of signaling networks, gene expression profiles and cell phenotyping, in combination with mathematical modeling, helped to understand the interface between growth factor and DNA signaling pathways in breast cancer [9]. According to the authors, the identification of rewiring patterns in apoptotic signaling pathways may have main implications for combined therapies in the treatment of breast cancer.

In this context, the main goal of this work was to profile the secretome composition of a murine cellular melanoma model composed by a normal melanocyte cell line, Melan-a, and its tumoral phenotype, Tm1 [10], using proteomic data to build interactomes for both secretomes. Our data revealed an expressive rewiring in the tumoral interactome of secreted proteins with important biological roles in malignant transformation including the transforming growth factor beta (TGF- $\beta$ ) signaling pathway.

\section{Material and methods}

\subsection{Cell culture and secretome harvesting}

Melan-a, an immortal line of pigmented melanocytes was derived from normal epidermal melanoblasts from embryos of inbred C57BL mice as earlier described [11]. Tm1 cell line was established after submitting Melan-a cells to sequential cycles of forced anchorage impediment, as reported by Oba-Shijno [10]. Melan-a cell line was used at passage 32 whereas $\operatorname{Tm} 1$ was used at passage 27. Cell lines were tested for mycoplasma infection and cultured as described previously [12]. Briefly, Melan-a, a murine melanocyte and Tm1, its transformedphenotype [10], were cultured in Roswell Park Memorial Institute (RPMI) medium ( $\mathrm{pH} 7.0$; Thermo Fisher, USA), containing $2.0 \mathrm{~g} / \mathrm{L}$ of sodium bicarbonate, $100 \mathrm{mg} / \mathrm{L}$ streptomycin, $25 \mathrm{mg} / \mathrm{L}$ ampicillin, $2 \mathrm{mM}$ glutamine, $10 \%$ of Fetal Bovine Serum (FBS) and $200 \mathrm{nM} 12$-o-tetradecanoyl phorbol-13-acetate (PMA; Sigma, USA). While the addition of PMA is required for the growth of Melan-a cells, the growth of Tm1 is not dependent on this tumor promoter nor it is toxic for these cells. Therefore, in order to obtain comparable conditions for cell culture and secretome harvesting, we used 200 nM PMA in the cell culture media for both Melan-a and Tm1 cells. Cell cultures were maintained at $37^{\circ} \mathrm{C}$ in a humidified atmosphere of 5\% CO2 and 95\% air. Sub confluent cell cultures were washed three-times with warm buffered phosphate-saline (without $\mathrm{Ca}^{2+}$ and $\mathrm{Mg}^{2+}$ ) and, after culturing the cells in $\mathrm{T} 175 \mathrm{~cm}^{2}$ flasks for $24 \mathrm{~h}$ under phenol red-free, FBS-free RPMI medium, the conditioned media was harvested and centrifuged $\left(2200 \mathrm{xg}, 10 \mathrm{~min}, 4^{\circ} \mathrm{C}\right)$ for removing any remaining cell. Protease inhibitor cocktail (SIGMAFAST ${ }^{\mathrm{TM}}$, Sigma, USA) was added to each sample and the secretomes were filtered $\left(0.22 \mu \mathrm{m}\right.$; Millipore, USA) and concentrated $\left(5000 \times g ; 4{ }^{\circ} \mathrm{C}\right)$ using ultrafiltration devices (Vivaspin 20, $3 \mathrm{kDa}$ cutoff, GE Healthcare, USA). Protein content were measured by the Bradford method [13]. Secretomes were independently obtained from two biological replicates derived from each cell line. At the time of each secretome collection cell viability was measured by dye-exclusion analysis (trypan blue).

\subsection{SDS-polyacrylamide gel electrophoresis and Western blot analysis}

SDS-PAGE was carried out according to Laemmli [14] and proteins were subjected to Western blotting analysis as described elsewhere [15] using anti-TGF $\beta$-3 rabbit polyclonal antibody (Thermo Fisher Scientific, USA, cat \# PA5-51070).

\subsection{In-solution trypsin digestion}

The in-solution trypsin digestion was performed according to the protocol described by Kleifeld et al. [16] with slight modifications. Briefly, a solution of $6 \mathrm{M}$ guanidine hydrochloride (GuHCl) was added to a sample of $100 \mu \mathrm{g}$ of protein from each secretome to a final concentration of $3 \mathrm{M} \mathrm{GuHCl}$, followed by the addition of $5 \mathrm{mM}$ dithiothreitol (DTT) (final concentration). The mixture was incubated at $65^{\circ} \mathrm{C}$ for $60 \mathrm{~min}$. Iodoacetamide (IAA) was then added to a final concentration of $15 \mathrm{mM}$ and the samples were incubated for $60 \mathrm{~min}$ at room temperature, in the dark. To quench the excess of IAA, DTT was added to a final concentration of $15 \mathrm{mM}$. Clean-up of samples was performed by the addition of ice-cold acetone ( 8 volumes) and methanol (1 volume), followed by the incubation of samples for $3 \mathrm{~h}$ at $-80^{\circ} \mathrm{C}$. After centrifugation at $14,000 \mathrm{xg}$ for $10 \mathrm{~min}$, protein pellets were washed twice with one volume of ice cold methanol and then resolubilized with $\mathrm{NaOH}$ solution (final concentration of $2.5 \mathrm{mM}$ ), followed by the addition of $50 \mathrm{mM}$ HEPES buffer, pH 7.5, to a final volume of $100 \mu \mathrm{L}$. Trypsin (Proteomics grade; Sigma, USA) was added at 1:100 ratio (enzyme/ substrate) and protein samples were incubated at $37^{\circ} \mathrm{C}$ for $18 \mathrm{~h}$. Tryptic peptides were desalted using C18 StageTips [17], dried in a SpeedVac and redissolved in $50 \mu \mathrm{L}$ of $0.1 \%$ formic acid prior to nanoflow liquid chromatography/tandem mass spectrometry (LC - MS/MS) analysis.

\subsection{Mass spectrometric analysis}

An aliquot (5 $\mu \mathrm{L}$, corresponding to $3 \mu \mathrm{g}$ of peptides) of the resulting peptide mixture was injected into a trap column packed with C18 $(100 \mu \mathrm{m}$ i.d. $\times 2 \mathrm{~cm})$ for desalting with $100 \%$ solvent A $(0.1 \%$ formic acid). Peptides were then eluted onto an analytical column (75 $\mu \mathrm{m}$ i.d. $\times$ $100 \mathrm{~mm}$ ) packed in house with Aqua ${ }^{\circledR} \mathrm{C}-185 \mu \mathrm{m}$ beads (Phenomenex, USA). Nanoflow liquid chromatography was performed on an Easy nanoLC system (Thermo Fisher Scientific, USA) coupled to an LTQOrbitrap Velos mass spectrometer (Thermo Fisher Scientific, USA). Peptides were loaded onto the column with solvent A ( $0.1 \%$ formic acid) and eluted with a linear 85 min gradient from 4 to $30 \%$ of solvent B $(0.1 \%$ formic acid in acetonitrile) at a flow rate of $200 \mathrm{~nL} / \mathrm{min}$. Spray voltage was set at $2.1 \mathrm{kV}, 200^{\circ} \mathrm{C}$ and the mass spectrometer was operated in data dependent mode, in which one full MS scan was acquired in the $m / z$ range of $400-1800$ followed by MS/MS acquisition using Collision-Induced Dissociation (CID) of the twelve most intense ions from the MS scan. MS spectra were acquired in the Orbitrap analyzer at 60,000 resolution (at $400 \mathrm{~m} / \mathrm{z}$ ). Dynamic exclusion was defined by a list size of 500 features and exclusion duration of $60 \mathrm{~s}$. For the survey (MS) scan Automatic Gain Control (AGC) target value of 1,000,000 was set whereas the AGC target value for the fragment ion (MS/MS) spectra was set to 10,000 ions. The lower threshold for targeting precursor ions in the MS scans was 3000 counts.

\subsection{Proteomics data processing}

Mass spectrometric (RAW) data were analyzed with MaxQuant software [18] (version 1.6.2.3). A False Discovery Rate (FDR) of $1 \%$ was 
required for both protein and peptide-to-spectrum match identifications. LTQ-Orbitrap Velos raw data were searched against a target database restricted to the taxonomy 'Rodentia' (UniProt/SwissProt release 2018_06; 25,190 entries). This database was also combined with the sequences of 245 common contaminants and concatenated with the reversed versions of all sequences. Enzyme specificity was set to trypsin and at least two missed cleavages were allowed; cysteine carbamidomethylation was selected as fixed modification whereas methionine oxidation, glutamine/asparagine deamidation and protein $\mathrm{N}$-terminal acetylation were selected as variable modifications. Peptide identification was based on a search with an initial mass deviation of the precursor ion of $7 \mathrm{ppm}$ and the fragment mass tolerance was set to 0.5 Da. Labelfree quantitation was performed using the MaxLFQ algorithm [19], with the 're-quantify' and 'match between runs' functions of MaxQuant software enabled. As is observed from complex proteomes such as those of vertebrates, peptides can be shared between homologous proteins or splice variants, leading to "protein groups". For each protein group in the MaxQuant's 'proteinGroups.txt' file, the first protein entry was selected as representative. The full list of MaxQuant parameters is presented in the Supplementary Table 1 . The mass spectrometry proteomics data have been deposited to the ProteomeXchange Consortium via the PRIDE partner repository [20] with the dataset identifier PXD015114.

\subsection{Bioinformatic analyses}

Protein identification was accepted after estimating the False Discovery Rate calculated by the Andromeda searching algorithm, in the MaxQuant environment. After filtering searching results with a False Discovery Rate $(\mathrm{FDR}) \leq 1 \%$, protein identifications were accepted if they contained at least two identified unique peptides. For each cell line secretome, only proteins/peptides identified/quantified in the two biological replicates were selected for further analysis. All the protein LFQ intensity values were $\log 2$-transformed in $\mathrm{R}$ scripting and statistical environment [21]. Statistical analyses were performed using the 'limma' package in R/Bioconductor [22,23]. After fitting a linear model to the data, an empirical Bayes moderated $t$-test was used for the comparisons and $P$ values were adjusted for multiple testing with the BenjaminiHochberg method. Proteins with an adjusted $P$-value $\leq 0.05$ and $\log _{2}(-$ fold change) $>1$ and $<-1$ were considered to be differentially expressed. Correlations between replicates were also calculated in $\mathrm{R}$, using Pearson correlation coefficient, and displayed as scatter plots. Gene ontology enrichment analysis was carried out using STRING platform [24] (https://string-db.org/); adjusted $p$-values $\leq 0.05$ were considered.

\subsection{Network analysis and community detection}

Identified proteins were manually annotated according to the information associated to their subcellular location available under gene ontology 'cellular component' category (GO-CC); only proteins which displayed GO-CC as secreted/extracellular and/or proteins that displayed annotated signal peptides were used for network analysis. The resulting protein lists for both cell lines were used to map proteinprotein interactions using the STRING public repository [24]. Minimum required interaction score was set to $\geq 0.7$ ('high-confidence interactions') when selecting edge lists for building each network. The 'igraph' R library [25] was used to create both networks (Melan-a and Tm1) using edge lists for each cell line interactome. Networks were further subjected to community detection as follows: the Melan-a network was subjected to the Louvain method for community detection [26] using the igraph package. The Louvain method finds communities in static networks by maximizing the so-called modularity measure [27]. After detecting the communities in Melan-a network, nodes in Tm1 not present in Melan-a were assigned to the communities, aiming at the modularity maximization, using an in-house Python script. A comparative analysis was performed between the Melan-a and Tm1 network partitions. The analysis considered the number of nodes and the local modularity of all communities from both networks to quantify the impact of the changes in the network structure. Each community was named after the node (protein) with the highest degree. Nodes were named according to their UniProt accession number and colored according to their attributes, namely 'exclusive' (i.e. proteins present in only one of the two networks), 'rewired' (proteins that had their location changed among the communities when comparing the two networks) and 'core' (proteins that remained in the same community when comparing both networks).

\section{Results and discussion}

\subsection{The secretome diversity of Melan-a and its transformed phenotype (Tm1) and their corresponding protein-protein interaction networks}

It is important to notice, that the cell growth in serum-free conditions might alter the composition of secreted proteins as a result of nutrient deprivation. Nevertheless, we observed that cells remained viable up to $48 \mathrm{~h}$ after FBS removal, with a significant decrease in viability after $36 \mathrm{~h}$ of serum starvation. Therefore, we chose to harvest the secretomes after $24 \mathrm{~h}$ of FBS removal. In fact, dye-exclusion assay showed that our experimental design for secretome harvesting did not significantly affect cell viability, which was found to be $\geq 95 \%$ in all biological replicates for both cell lines (97.8\% for Melan-a and $97.5 \%$ for Tm1).

Proteomic analysis of Melan-a and Tm1 secretomes resulted in the identification of 977 proteins (Supplementary Table 2), with good correlation for label-free quantitation between the two biological replicates for each cell line, as observed by their Pearson correlation coefficients $\geq 0.9$ (Fig. 1A). Both cell line secretomes displayed a number of exclusive proteins, being 75 exclusives in Melan-a and 217 exclusives in Tm1 (Fig. 1B); most of which (685) were shared between both cell line secretomes. The identification of proteins in both secretomes evidenced the presence of some intracellular proteins (Supplementary Table 2). Although unconventional protein secretion may be correlated with the identification of intracellular proteins [28], apoptosis of serum-deprived cells may have led to residual contamination of the analyzed secretomes with such proteins.

The analysis of exclusive proteins in the $\operatorname{Tm} 1$ secretome revealed several cancer-related proteins including an endoglycosidase heparanase (Supplementary Table 2). Actually, the expression of heparanase is increased not only in Tm1 but also in the other cell lineages derived from the nontumorigenic cell line Melan-a and such endoglycosidase may likely contribute to early changes in malignant transformation [10]. In addition to its involvement in the degradation and remodeling of extracellular matrix, heparanase is a positive regulator of protein kinase B signaling, a classic pathway which is constitutively activated in melanoma [29]. Since proteolytic signaling is significantly related to several oncogenic events, our group have recently profiled Melan-a and $\mathrm{Tm} 1$ secretomes, focusing on the proteolytic events in both secretomes [12]. Even though in our previous work both secretomes were found enriched in protease-generated protein fragments, through the use of Terminal Amine Isobaric Labeling of Substrates (TAILS), some cancerrelated proteins were found only in the tumoral (Tm1) secretome.

Statistical analysis (eBayes moderated $t$-test) performed on the 685 shared proteins revealed that both secretomes displayed a number of differentially expressed proteins, with processes related to multicellular organism development enriched in the set of differentially expressed proteins in Melan-a secretome and biosynthetic processes enriched in Tm1 secretome (Fig. 1C and D and Supplementary Table 3). In fact, biosynthetic processes such as translation demand a substantial energy input; this is a predictable feature in rapidly growing melanoma cells [30] and is in line with the data derived from growth curve of Tm1 cell lines, which displayed shorter doubling times $(21 \mathrm{~h})$ in comparison to the normal counterpart, Melan-a $(26 \mathrm{~h})$. Interestingly, some of the significantly up-regulated proteins in $\mathrm{Tm} 1$ secretome were somehow 
A
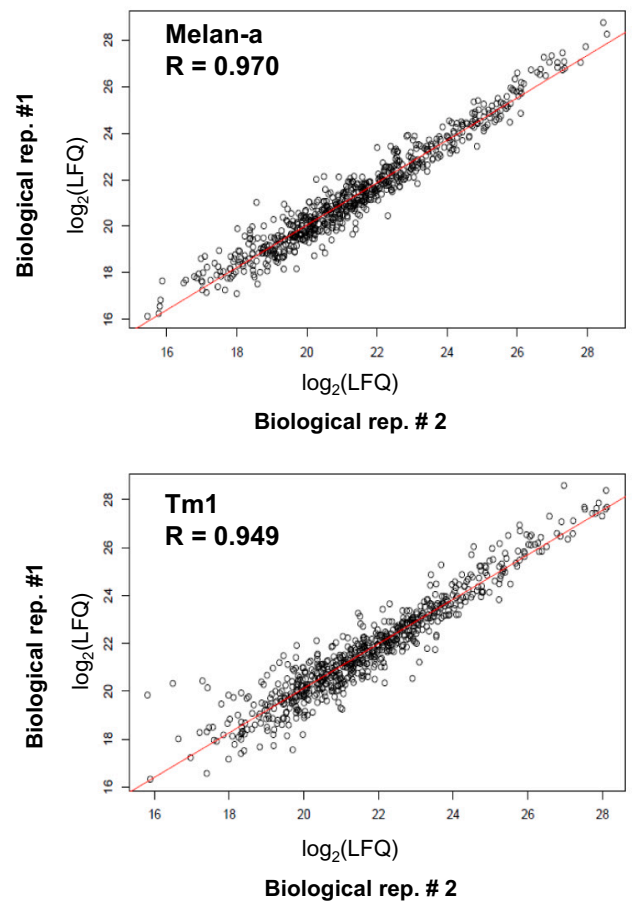

B
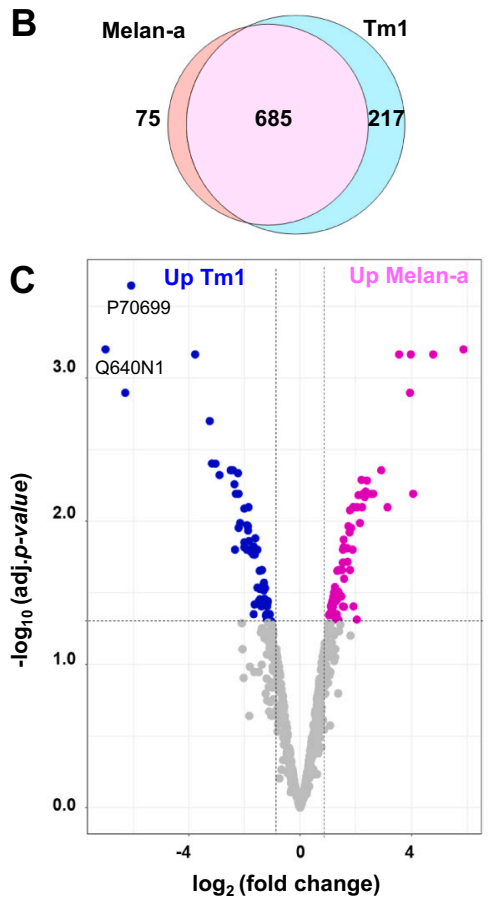

D

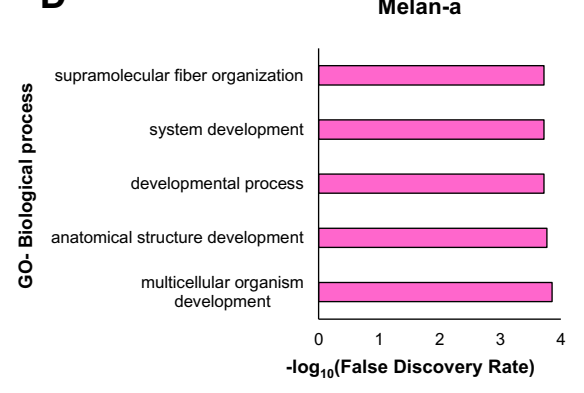

$\operatorname{Tm} 1$

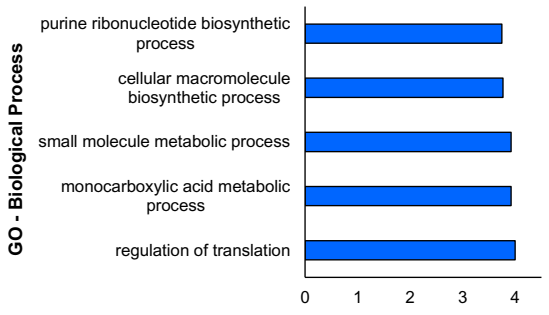

$-\log _{10}$ (False Discovery Rate)

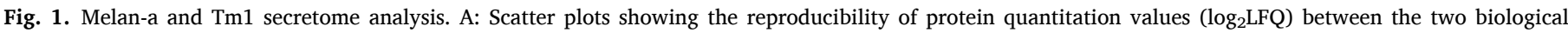

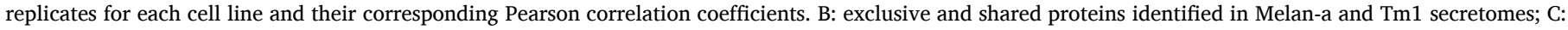

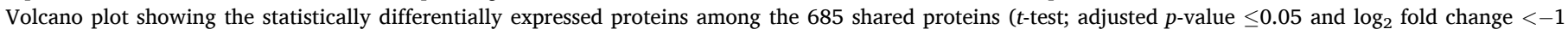

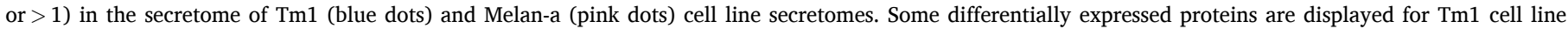

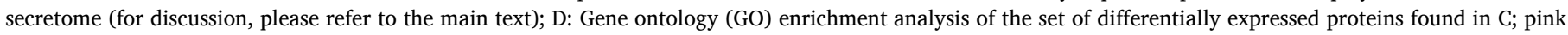

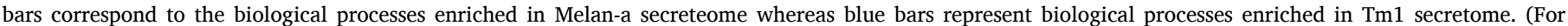
interpretation of the references to colour in this figure legend, the reader is referred to the web version of this article.)

correlated with the process of malignant transformation. This was the case for adipocyte enhancer-binding protein 1 (AEBP1; $\log _{2}$ (fold change $=-6.9)$ ) and Lysosomal alpha-glucosidase (GAA; $\log _{2}$ (fold change $=-6.0)$ ), which were among the differentially expressed proteins in Tm1 secretome. AEBP1 is a positive regulator of collagen fibrillogenesis, involved in the organization and remodeling of the extracellular matrix [31]. Moreover, upregulation of AEBP1 confers acquired resistance to BRAF (V600E) inhibition in melanoma and may represent a novel therapeutic target for treating BRAF inhibitor-resistant melanoma [32]. Lysosomal alpha-glucosidase is an essential enzyme involved in glycogen catabolic process in lysosomes [33] which, in turn, releases glucose that may eventually be used to fulfill the increased energy demand in rapidly growing cells during malignant transformation.

In order to further evaluate the contribution of both secretomes to the functional phenotype of each cell line, we created two networks of protein-protein interactions based on annotations available at the STRING public repository [24]. The rationale underlying this analysis was to use the list of identified proteins from both cell line secretomes to build two interaction networks (normal and tumoral networks, respectively) and to identify clusters of interactions (communities) which may eventually have functional relevance in the context of tumorigenesis. For this purpose, the list of identified proteins in both secretomes was subjected to a filtering step, selecting only proteins whose (i) GO annotations (cellular component) were referred to be 'secreted/extracellular' and/or (ii) proteins that displayed annotated signal peptides. Protein in the resulting list (290 and 331 unique proteins in Melan-a and Tm1, respectively) had their interactions mapped using the STRING public repository. We defined as protein-protein interaction any curated association among proteins that had been mapped by functional/ descriptive experiments such as co-expression, co-occurring proteins or gene fusion assays. Therefore, only high-confidence interactions (i.e. minimum required interaction score $\geq 0.7$ ) were selected for building the interaction networks. This final filtering step resulted in two networks comprising 181 and 212 unique proteins for Melan-a and Tm1, respectively. Although the filtering steps have lowered considerably the number of proteins in both networks, we decided to keep such conservative approach to avoid potentially spurious interactions derived from a non-curated protein list. Finally, the networks were subjected to community detection using an in-house Python script which basically find communities by maximizing a quality measure, called modularity. Overall, the communities of both networks shared several nodes, however differences were noticed within each network, such as exclusive and rewired nodes (Supplementary Table 4). Although some overlapping might exist between the nodes within each community in the normal and tumoral networks, the overall topology reflects the connectivity pattern of each node with its neighbors. We named the communities by the UniProt accession number of the node with the highest degree. Topological measures for both networks are presented in

Table 1

Topology metrics for both Melan-a (normal) and Tm1 (tumoral phenotype) networks.

\begin{tabular}{lll}
\hline Measure & Melan-a & Tm1 \\
\hline \# of Nodes & 181 & 212 \\
\# of Edges & 771 & 907 \\
\# of communities & 13 & 15 \\
Average degree & 8.52 & 8.56 \\
Max. Degree & 43 & 49 \\
Min. Degree & 1 & 1 \\
\hline
\end{tabular}


Table 1.

Although the tumoral protein-protein interaction network displayed a slightly higher number of interactions and communities than the normal phenotype network, no further differences were noticed. Therefore, to gain insights into the network structures and the potential biological implications, we mapped the nodes that were present in both networks, considering those that remained in the same community (regardless of the cell line); these conserved nodes were named as 'core' nodes (Fig.2).

On the other hand, the presence of several exclusive proteins resulted in 'exclusive' nodes in each network. In addition, a number of nodes changed their location among the communities, and such proteins were referred to as 'rewired' nodes. These exclusive and rewired nodes were highlighted in Fig.2 (yellow and blue nodes, respectively) and although both networks presented such nodes, this number was expressively higher in the tumoral phenotype network (Fig. 3a). For example, the communities P98064 and Q62433 comprised only 'new' nodes and were exclusive for the tumoral network. Interestingly, these two proteins (P98064 and Q62433) have been implicated in oncogenesis, in distinct cancer types. High serum levels of the mannan-binding lectin serine protease 1 (MASP, P98064) have been associated with cervical cancer progression and worse disease prognosis [34]. Protein NDRG1 (Q62433) is a stress-responsive protein involved in hormone responses, cell growth and differentiation, acting as a tumor suppressor in many cell types [35]. Interestingly, although median expression values were similar for core and rewired nodes in both networks, exclusive nodes varied and the magnitude of this variation was higher in the tumoral network, as illustrated by the highest standard deviation in comparison to the other node types (Fig. 3b). In addition, such higher standard deviation did not arise from sparse data, since the number of exclusive nodes in Tm1 network was higher (50 nodes) than the number of exclusive nodes in Melan-a network (16 nodes); rather it is a result of a higher amplitude in protein expression values for the tumoral secretome.

Remarkably, some of the communities were expressively rewired in the $\operatorname{Tm} 1$ network, leading to the increment of the number of nodes. In this context, we noticed that the community P11276 displayed the highest number of exclusive nodes in the tumoral phenotype, resulting in a significant change in the connectivity within this community compared to the normal counterpart (Fig.4a). Fibronectin (P11276) is an extracellular matrix protein, whose up-regulation is related to TGF $\beta$ activity; it is highly expressed by several cancer cells and with key roles in tumorigenesis, acting as a positive regulator in cell migration, for example [36]. Gene ontology analysis of the exclusive nodes of this community revealed the enrichment of important signaling pathways related to oncogenesis, such as the transmembrane receptor protein serine/threonine kinase signaling and transforming growth factor beta receptor signaling pathway (TGF $\beta$ )(Fig. 4b); therefore, we referred to these nodes as a 'cancer-related' core. Differently, in this same community biological processes related to cellular component organization were enriched in the set of the core nodes (Fig. 4c).

In order to further evaluate the contribution of secreted proteins in normal and tumoral phenotype for the enriched processes related to TGF $\beta$ signaling pathway we profiled both normal and tumoral secretomes for the expression of TGF $\beta$-3, one of the three mammalian isoforms of this growth factor whose increased expression level is associated to melanoma progression [37]. A major protein band at approximately $55 \mathrm{kDa}$ was detected in the $\mathrm{Tm} 1$ secretome in Western blot analysis, suggesting a higher level of the protein precursor of TGF $\beta$ 3 (pro-TGF $\beta$ ) in the tumoral secretome compared to the normal phenotype, Melan-a, in which a faint protein band was observed (Fig. 4d). Furthermore, it is possible to suggest that a small portion of this cytokine was present in a processed form in the tumoral secretome, since a protein band at lower molecular mass $(\sim 31 \mathrm{kDa})$ was also detected.

\subsection{Emerging patterns in melanoma interactome: insights into the functional significance for the tumoral phenotype}

From the molecular point of view, melanoma is characterized by constitutive activation of signaling pathways related to the cell cycle, such as mitogen-activated protein kinase (MAPK-) and phosphoinositide 3-kinase (PI3K)-signaling pathways, driven by mutant BRAF or NRAS
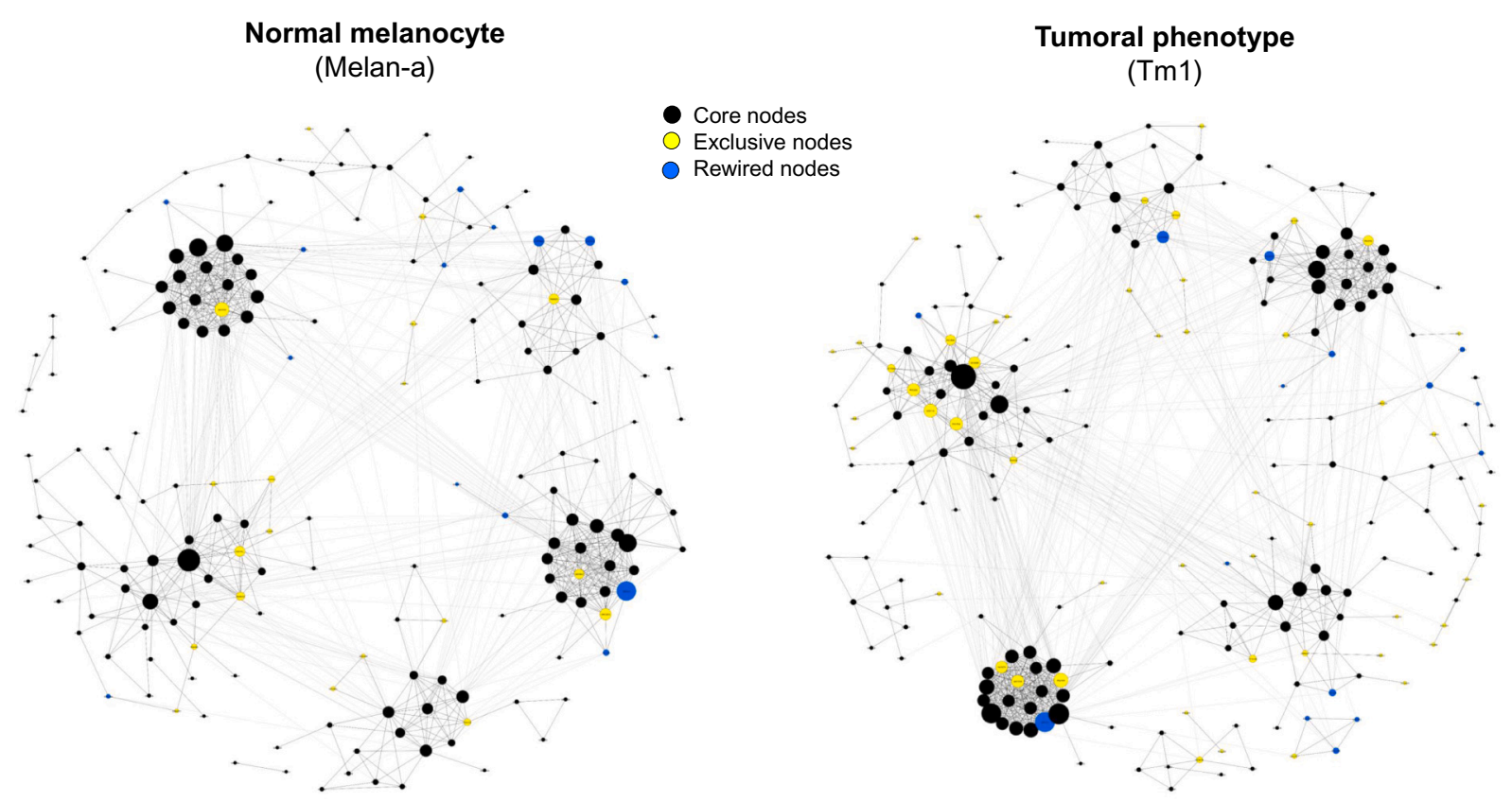

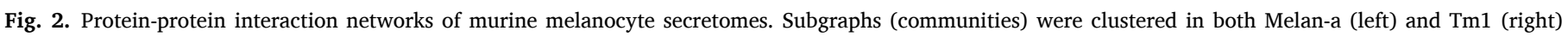

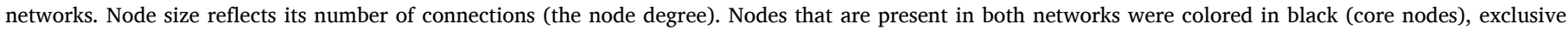

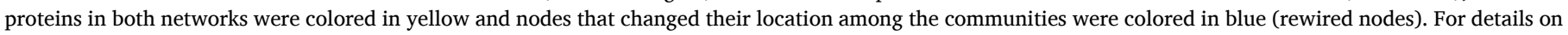

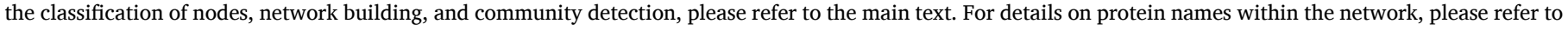
supplementary Table 4. (For interpretation of the references to colour in this figure legend, the reader is referred to the web version of this article.) 
A

Normal melanocyte

(Melan-a)

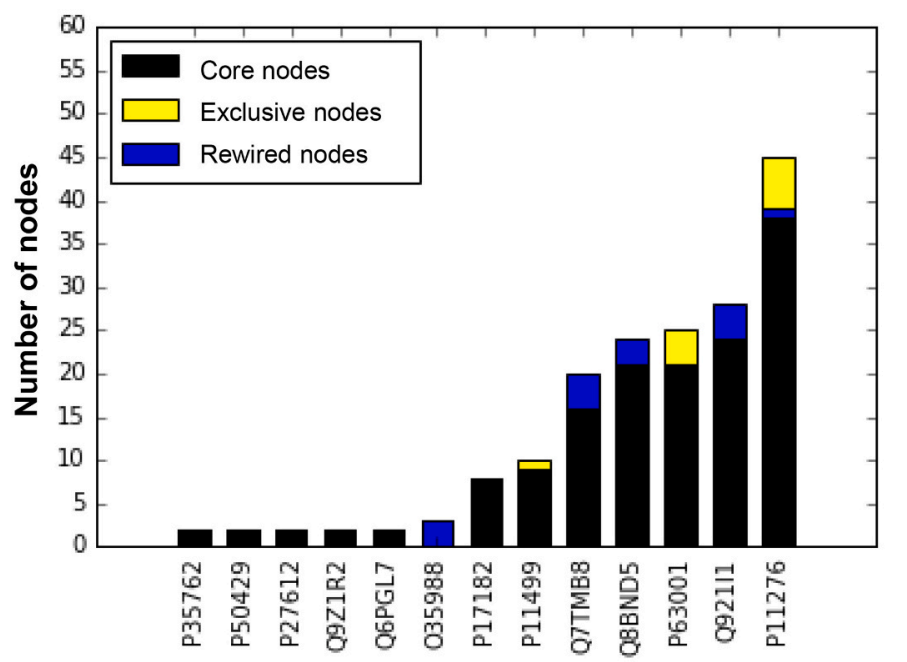

Community

B

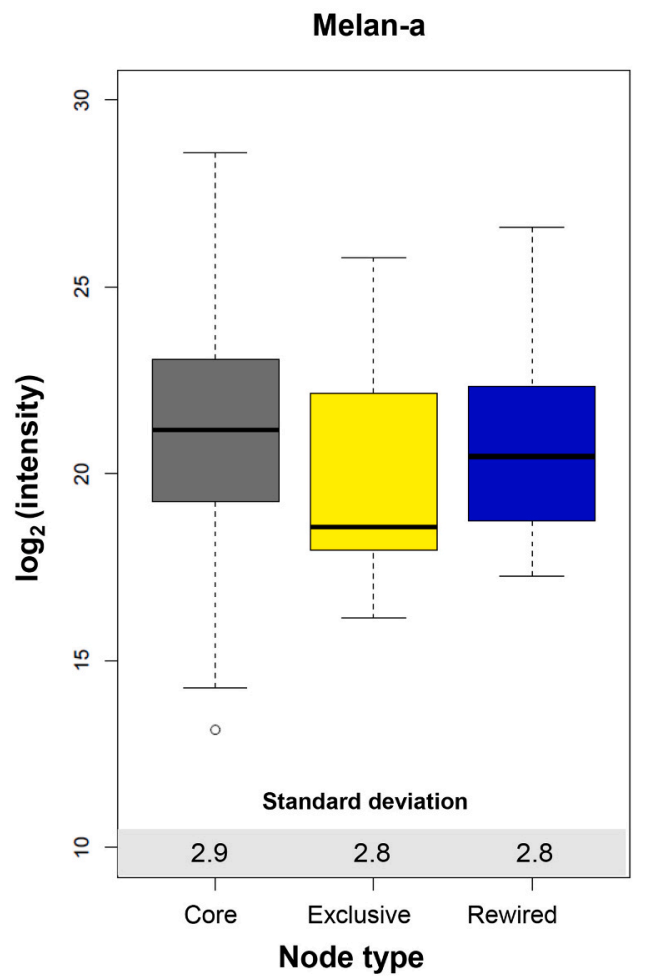

Tumoral phenotype

(Tm1)

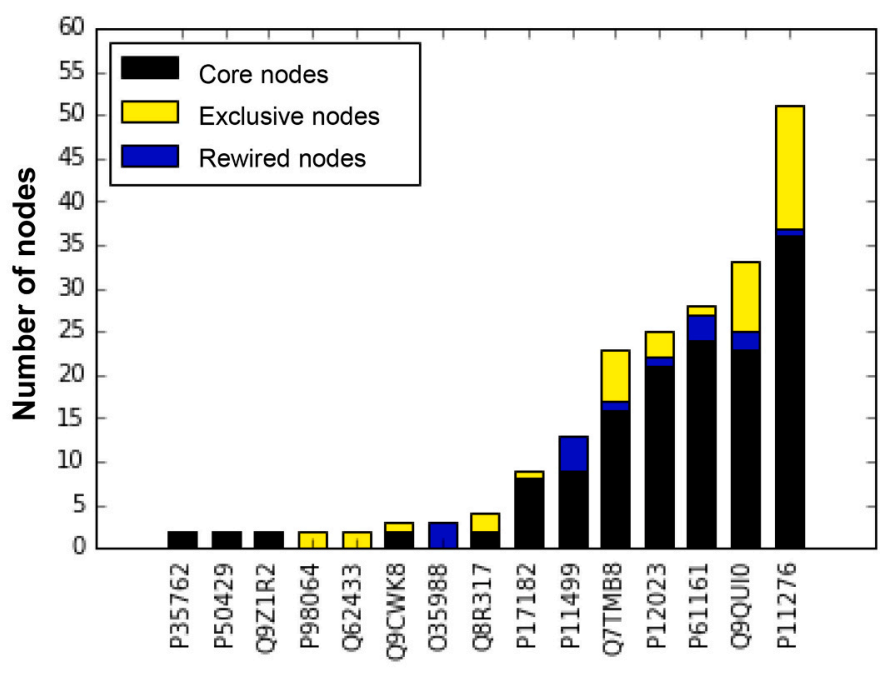

Community

Tm1

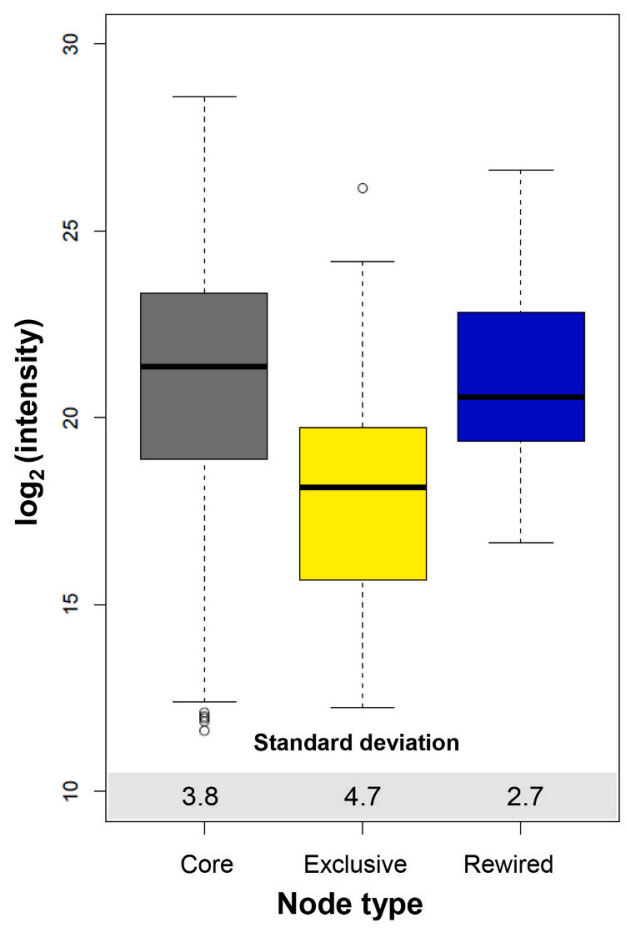

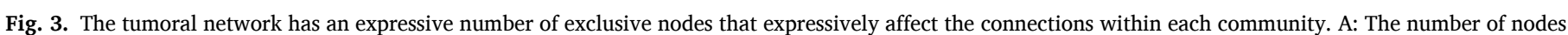

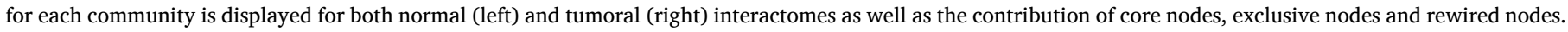

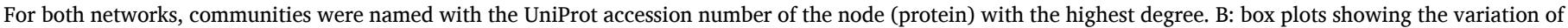
expression values ( $\log _{2}$ of normalized intensity values) among all node types in both networks.

genes $[29,38]$. Although these signaling networks take place intracellularly, they are both activated by interaction among tyrosine kinase receptors and secreted soluble factors. Among these factors, TGF $\beta$ has been implicated in melanoma progression, invasion and metastasis $[37,39,40]$. Indeed, the tumoral secretome displayed significant interactions among proteins participating in TGF $\beta$ signaling interaction network, which may have potential implications for melanoma progression. A major example of such emerging pattern was found in P11276 community, in which exclusive nodes significantly contributed to the enrichment of biological processes related to TGF $\beta$ signaling (Fig. 3a). Such enrichment resulted from the presence of some proteins related to the TGF $\beta$ signaling network, including latent-transforming growth factor beta-binding proteins, besides TGF $\beta$ itself (Supplementary Table 5). In this way, our group has recently reported on the non- 
A

Tumoral
phenotype
(Tm1)
Community
P11276
Core nodes
Exclusive nodes
Rewired nodes

D
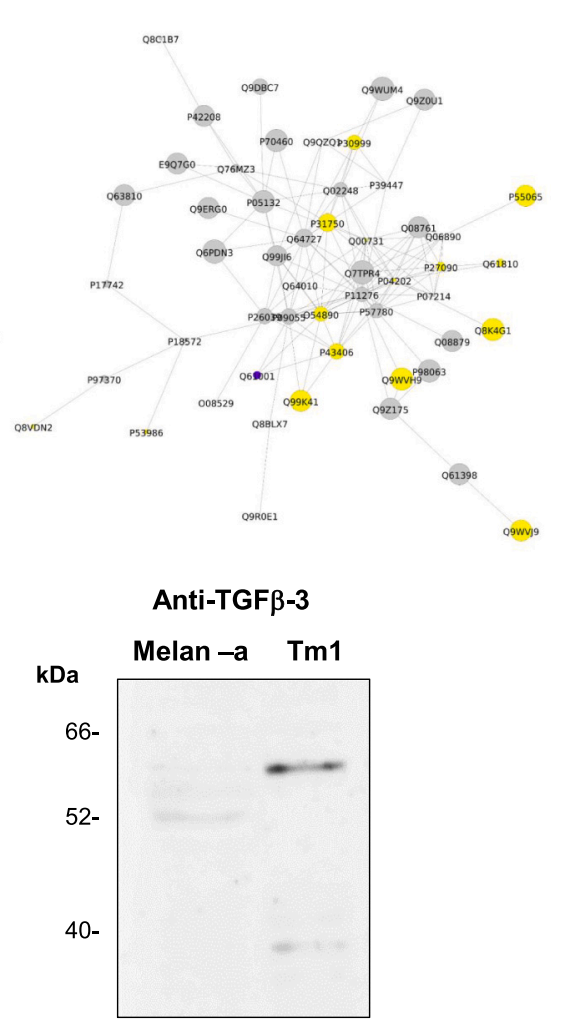

B Exclusive nodes (cancer-related core)

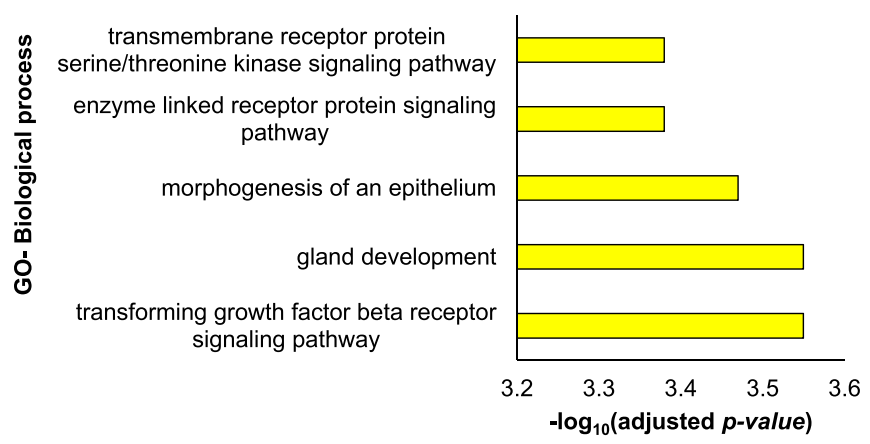

\section{Core nodes}

C

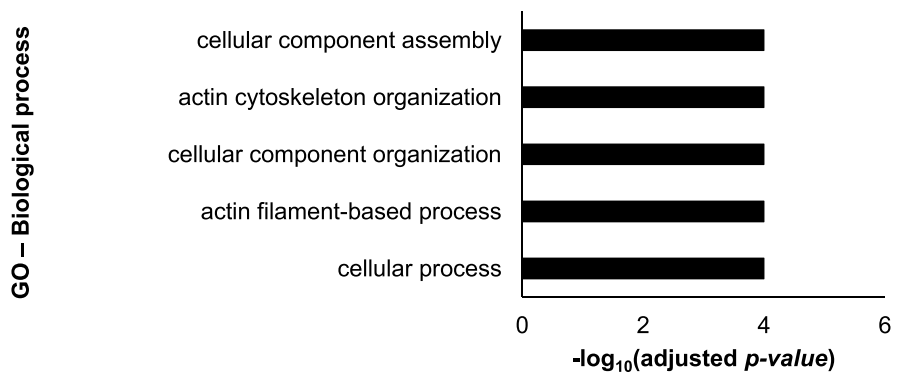

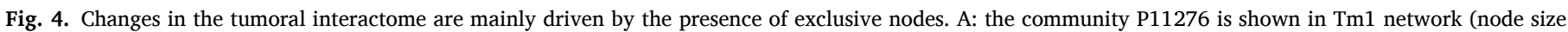

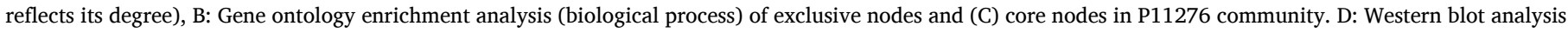

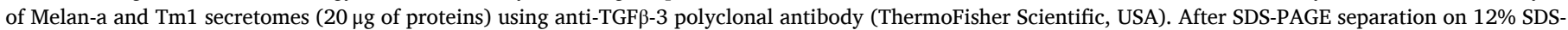

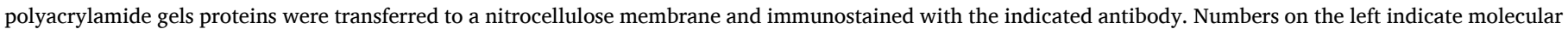
mass marker mobility.

canonical proteolytic processing of some growth factors, including $\mathrm{TGF} \beta-3$, in the Tm1 secretome through the use of proteomics/Nterminomics analyses [12]. Mass spectrometry-based analysis suggested the proteolytic processing of TGF $\beta-3$ in a position upstream its canonical proteolytic processing site. Such non-canonical processed form of TGF $\beta-3$ would, therefore, have a higher molecular mass when compared to the canonical monomeric form $(\sim 13 \mathrm{kDa})$. Moreover, in addition to the $\sim 55 \mathrm{kDa}$ protein band, which likely represents the proTGF $\beta$, our Western blot analysis showed a protein band at nearly $31 \mathrm{kDa}$ that might correspond to a non-canonical processed form of TGF $\beta-3$. In fact, both standard (trypsin-specific) and semi-specific mass spectrometry database searches resulted in peptides that matched to the proforms of TGF $\beta$ (i.e. the latency-associated peptide; Supplementary Table 6); therefore, this result is in line with the hypothesis of noncanonical proteolytic processing of this cytokine.

The processing of TGF $\beta$ has been investigated and some diverse proteolytic processed forms have been described so far [41,42] [43],. In fact, accumulated evidence has indicated that pro-TGF $\beta$ may be secreted and proteolytically processed in the extracellular environment $[44,45]$. The biological roles of such non-canonical proteolytic processed forms remain to be evaluated. Interestingly, TGF $\beta$ is involved in a number of signaling processes that result in the synthesis of many of its own activators, such as latent-transforming growth factor beta-binding proteins, integrins and proteases [46]. In line with these findings, the mapping and annotation of cleavage sites in Tm1 secretome suggested the involvement of active proteases in the proteolytic processing of growth factors, including TGF $\beta$ [12]. Indeed, TGF $\beta$ signaling network is a promising pathway for pharmacological intervention in melanoma cases, since it has been implicated in the adaptive resistance to BRAF (V600E) inhibition [40].

Functional enrichment analysis of core nodes revealed conserved biological functions for these nodes in both networks. (Supplementary fig. 1). Therefore, core nodes might represent proteins whose biological roles are essential for cell survival, regardless of the transformed phenotype. Conversely, when considering only the exclusive nodes in the tumoral phenotype network, some cancer-related processes where found to be enriched, such as phosphorylation processes, cell division and cyclin turnover, for example. In addition, when comparing the two networks (the normal and the tumoral phenotypes) it was possible to track the rewired pattern of selected nodes within each network. The presence of exclusive nodes and, more specifically, cancer-related cores, have main consequences for network rewiring, resulting in new connections among the observed interactomes. Thus, our data suggest that the shift in the tumoral interactome of secreted proteins results in an emerging (rewired) pattern of protein-protein interaction network which is mainly driven by the presence of exclusive nodes. Rewiring connections among biological signaling circuits represent a parsimonious scenario in cancer metabolic pathways, when nutrient and oxygen are scarce $[47,48]$. In this context, our findings suggest that the rearrangement in the tumoral interactome may be correlated with the malignant transformation of melanocytes associated with substrate adhesion impediment. Whether the tumoral interactome of secreted proteins is more effective in targeting biological pathways related to melanoma progression is a feature that remains to be explored.

Recent data on network-based prediction of protein interactions shed light on the underlying biological basis of interactomes [49]. According to the authors, structural and evolutionary evidences suggest that proteins do not interact if they are similar, rather the connectivity is a result of the similarity of a given protein to the partners of its interactor. In fact, our results also point into this direction since, as illustrated by the GO enrichment analysis of the communities in both networks, functionally related clusters were present in both networks (Supplementary 
fig. 1). Collectively, given the molecular heterogeneity of melanoma our results partially underscore the complex cellular logic of melanoma interactome and point out to a versatile way of the diversification of protein-protein interactions during oncogenesis. Since cancer-related cores might be associated to neoplastic conversion, the tumoral secretome may likely affect autocrine and paracrine signaling in melanoma. We have recently shown, with a heterotypic signaling cellular model in human melanoma, that self- and cross-stimulation may play a key role in shaping the tumor microenvironment and enable tumoral cells to succeed in the process of melanoma progression and metastasis [50].

Nonetheless, cellular crosstalk is an important feature of biological systems, modifying the repertoire of secreted proteins in malignant cells as well as in stromal ones. In this context, although protein-protein interaction data used in this work were derived from curated repositories, the interactomes reported herein may lack the full potential interactors found in a more complex environment (i.e. a normal tissue and its tumoral counterpart) since our work was carried out using secretomes derived from cultured cell lines. Therefore, the communities detected in our work may represent biological patterns of an even more complex interactome found in the tumoral environment.

\section{Concluding remarks}

In general, proteomics experiments aiming to identify differentially expressed proteins end up with a list of proteins whose expression values are somewhat statically significant. Usually, the next step is a functional enrichment analysis, to verify the overrepresentation of certain GO terms, adding biological significance to the set of differentially expressed proteins. Therefore, the biological assumptions are often made at the level of individual proteins and their contribution to the observed phenotype. In this context, it was recently demonstrated that pathway enrichment analysis may perform sub optimally when proteins are implicated in multiple biological pathways [51]. Therefore, functional network community detection can disaggregate and filter multiple underlying pathways in enrichment analyses. Indeed, our dataset illustrates this situation: statistically significant differentially expressed proteins showed the enrichment of a few biological pathways between the normal and tumoral phenotype. Moreover, proteins whose expression levels did not result in statistically significant differences between the two phenotypes may be worthy of investigation in the context of oncogenesis. In fact, the identification of unique proteins or differential protein expression patterns per se, did not allow for a comprehensive understanding of the biological complexity underlying the normal and tumoral phenotypes; rather, when interactomes were assembled and, more importantly, when communities were identified, some emerging patterns were observed. Finally, these subgraphs might represent functional network motifs, with constitutive (core nodes) and/or adaptative roles (new/rewired nodes) whose interactions (if conserved in homologous organisms, such as humans) could potentially be targeted for therapeutic intervention in melanoma treatment.

Supplementary data to this article can be found online at https://doi. org/10.1016/j.jprot.2020.104063.

\section{Declaration of Competing Interest}

The authors declare no conflict of interest.

\section{Data availability}

The mass spectrometry proteomics data have been deposited to the ProteomeXchange Consortium via the PRIDE partner repository with the dataset identifier PXD015114.

\section{Acknowledgments}

This work was supported by grants from Fundação de Amparo à
Pesquisa do Estado de São Paulo (FAPESP, grants \# 2013/07467-1; 2014/06579-3; 2017/22330-3; 2017/24185-0, 2015/21660-4, 2013/ 07375-0; 2019/10817-0).

\section{Author contributions}

R.F analyzed the data and wrote the paper, R.B and D.S.P performed cell culture experiments and proteomics workbench, D.A.S performed MS analysis, M.F.C and U.B collected the secretomes and performed the Western blotting experiments; S.M.T.S, M.C.V.N, R.C and S.G.S analyzed the data and wrote the paper; A.Z analyzed the data, wrote the paper and designed the experiments. All authors reviewed the manuscript.

\section{References}

[1] J.L. Paltridge, L. Belle, Y. Khew-Goodall, The secretome in cancer progression, Biochim. Biophys. Acta - Proteins Proteomics. 1834 (2013) 2233-2241, https:// doi.org/10.1016/j.bbapap.2013.03.014.

[2] L. Kuhlmann, E. Cummins, I. Samudio, T. Kislinger, Cell-surface proteomics for the identification of novel therapeutic targets in cancer, Expert Rev. Proteomics. 15 (2018) 259-275, https://doi.org/10.1080/14789450.2018.1429924.

[3] D. Hanahan, R.A. Weinberg, Hallmarks of cancer: the next generation, Cell. 144 (2011) 646-674, https://doi.org/10.1016/j.cell.2011.02.013.

[4] P. Creixell, J. Reimand, S. Haider, G. Wu, T. Shibata, M. Vazquez, V. Mustonen, A. Gonzalez-Perez, J. Pearson, C. Sander, B.J. Raphael, D.S. Marks, B.F.F. Ouellette, A. Valencia, G.D. Bader, P.C. Boutros, J.M. Stuart, R. Linding, N. Lopez-Bigas, L. D. Stein, Pathway and network analysis of cancer genomes, Nat. Methods 12 (2015) 615-621, https://doi.org/10.1038/nmeth.3440.

[5] T.Y. Kim, H.U. Kim, S.Y. Lee, Data integration and analysis of biological networks, Curr. Opin. Biotechnol. 21 (2010) 78-84, https://doi.org/10.1016/j. copbio.2010.01.003.

[6] S. Zhang, G. Jin, X.S. Zhang, L. Chen, Discovering functions and revealing mechanisms at molecular level from biological networks, Proteomics. 7 (2007) 2856-2869, https://doi.org/10.1002/pmic.200700095.

[7] A. Kaushik, Y. Bhatia, S. Ali, D. Gupta, Gene network rewiring to study melanoma stage progression and elements essential for driving melanoma, PLoS One 10 (2015), https://doi.org/10.1371/journal.pone.0142443.

[8] Z.J. Cui, X.H. Zhou, H.Y. Zhang, DNA methylation module network-based prognosis and molecular typing of cancer, Genes (Basel) 10 (2019) 571, https:// doi.org/10.3390/genes10080571.

[9] M.J. Lee, A.S. Ye, A.K. Gardino, A.M. Heijink, P.K. Sorger, G. MacBeath, M.B. Yaffe, Sequential application of anticancer drugs enhances cell death by rewiring apoptotic signaling networks, Cell. 149 (2012) 780-794, https://doi.org/10.1016/ j.cell.2012.03.031.

[10] S.M. Oba-Shinjo, M. Correa, T.I. Ricca, F. Molognoni, M.A. Pinhal, I.A. Neves, S. K. Marie, L.O. Sampaio, H.B. Nader, R. Chammas, M.G. Jasiulionis, Melanocyte transformation associated with substrate adhesion impediment, Neoplasia. 8 (2006) 231-241, https://doi.org/10.1593/neo.05781.

[11] D.C. Bennett, P.J. Cooper, I.R. Hart, A line of non-tumorigenic mouse melanocytes, syngeneic with the B16 melanoma and requiring a tumour promoter for growth, Int. J. Cancer 39 (1987) 414-418, https://doi.org/10.1002/ijc.2910390324.

[12] T. Liberato, I. Fukushima, E.S. Kitano, S.M.T. Serrano, R. Chammas, A. Zelanis, Proteomic profiling of the proteolytic events in the secretome of the transformed phenotype of melanocyte-derived cells using Terminal Amine Isotopic Labeling of Substrates, J. Proteome 192 (2019) 291-298, https://doi.org/10.1016/j. jprot.2018.09.010.

[13] M.M. Bradford, A rapid and sensitive method for the quantitation of microgram quantities of protein utilizing the principle of protein-dye binding, Anal. Biochem. 72 (1976) 248-254, https://doi.org/10.1016/0003-2697(76)90527-3.

[14] U.K. Laemmli, Cleavage of structural proteins during the assembly of the head of bacteriophage T4, Nature. 227 (1970) 680-685, https://doi.org/10.1038/ 227680a0.

[15] H. Towbin, T. Staehelin, J. Gordon, Electrophoretic transfer of proteins from polyacrylamide gels to nitrocellulose sheets: procedure and some applications, Proc. Natl. Acad. Sci. U. S. A. 76 (1979) 4350-4354, https://doi.org/10.1073/ pnas.76.9.4350.

[16] O. Kleifeld, A. Doucet, A. Prudova, U. Auf Dem Keller, M. Gioia, J. N. Kizhakkedathu, C.M. Overall, Identifying and quantifying proteolytic events and the natural $\mathrm{N}$ terminome by terminal amine isotopic labeling of substrates, Nat. Protoc. 6 (2011) 1578-1611, https://doi.org/10.1038/nprot.2011.382.

[17] J. Rappsilber, M. Mann, Y. Ishihama, Protocol for micro-purification, enrichment, pre-fractionation and storage of peptides for proteomics using StageTips, Nat. Protoc. 2 (2007) 1896-1906, https://doi.org/10.1038/nprot.2007.261.

[18] J. Cox, M. Mann, MaxQuant enables high peptide identification rates, individualized p.p.b.-range mass accuracies and proteome-wide protein quantification, Nat. Biotechnol. 26 (2008) 1367-1372, https://doi.org/10.1038/ nbt.1511.

[19] J. Cox, M.Y. Hein, C.A. Luber, I. Paron, N. Nagaraj, M. Mann, Accurate proteomewide label-free quantification by delayed normalization and maximal peptide ratio extraction, termed MaxLFQ, Mol. Cell. Proteomics 13 (2014) 2513-2526, https:// doi.org/10.1074/mcp.m113.031591. 
[20] J.A. Vizcaíno, E.W. Deutsch, R. Wang, A. Csordas, F. Reisinger, D. Ríos, J. A. Dianes, Z. Sun, T. Farrah, N. Bandeira, P.A. Binz, I. Xenarios, M. Eisenacher, G. Mayer, L. Gatto, A. Campos, R.J. Chalkley, H.J. Kraus, J.P. Albar, S. Martinez Bartolomé, R. Apweiler, G.S. Omenn, L. Martens, A.R. Jones, H. Hermjakob, ProteomeXchange provides globally coordinated proteomics data submission and dissemination, Nat. Biotechnol. 32 (2014) 223-226, https://doi.org/10.1038/ nbt. 2839 .

[21] I. Ross, G. Robert, R. Ihaka, R. Gentleman, R: a language for data analysis and graphics, J. Comput. Graph. Stat. 5 (1996) 299-314, https://doi.org/10.1080/ 10618600.1996.10474713.

[22] R. Gentleman, V. Carey, D. Bates, B. Bolstad, M. Dettling, S. Dudoit, B. Ellis, L. Gautier, Y. Ge, J. Gentry, K. Hornik, T. Hothorn, W. Huber, S. Iacus, R. Irizarry, F. Leisch, C. Li, M. Maechler, A. Rossini, G. Sawitzki, C. Smith, G. Smyth, L. Tierney, J. Yang, J. Zhang, Bioconductor: open software development for computational biology and bioinformatics, Genome Biol. 5 (2004) R80, https:// doi.org/10.1186/gb-2004-5-10-r80.

[23] G.K. Smyth, Linear models and empirical Bayes methods for assessing differential expression in microarray experiments linear models and empirical Bayes methods for assessing differential expression in microarray experiments, Stat. Appl. Genet. Mol. Biol. 3 (2004) 1-26, https://doi.org/10.2202/1544-6115.1027.

[24] D. Szklarczyk, J.H. Morris, H. Cook, M. Kuhn, S. Wyder, M. Simonovic, A. Santos, N.T. Doncheva, A. Roth, P. Bork, L.J. Jensen, C. Von Mering, The STRING database in 2017: quality-controlled protein-protein association networks, made broadly accessible, Nucleic Acids Res. 45 (2017) D362-D368. doi:https://doi.org /10.1093/nar/gkw937.

[25] G. Csardi, T. Nepusz, The igraph Software Package for Complex Network Research, Int. J. Compl. Syst. (2006), 1695.

[26] V.D. Blondel, J.L. Guillaume, R. Lambiotte, E. Lefebvre, Fast unfolding of communities in large networks, J. Stat. Mech. Theory Exp. 2008 (2008), https:// doi.org/10.1088/1742-5468/2008/10/P10008.

[27] M.E. Newman, Modularity and community structure in Networks, Proc. Natl. Acad. Sci. (2009) 1-30.

[28] L. Villarreal, O. Méndez, C. Salvans, J. Gregori, J. Baselga, J. Villanueva, Unconventional secretion is a major contributor of Cancer cell line Secretomes, Mol. Cell. Proteomics 12 (2013) 1046-1060, https://doi.org/10.1074/mcp. M112.021618.

[29] B. Garman, I.N. Anastopoulos, C. Krepler, P. Brafford, K. Sproesser, Y. Jiang, B. Wubbenhorst, R. Amaravadi, J. Bennett, M. Beqiri, D. Elder, K.T. Flaherty, D. T. Frederick, T.C. Gangadhar, M. Guarino, D. Hoon, G. Karakousis, Q. Liu, N. Mitra, N.J. Petrelli, L. Schuchter, B. Shannan, C.L. Shields, J. Wargo, B. Wenz, M. A. Wilson, M. Xiao, W. Xu, X. Xu, X. Yin, N.R. Zhang, M.A. Davies, M. Herlyn, K. L. Nathanson, Genetic and genomic characterization of 462 melanoma patientderived Xenografts, tumor biopsies, and cell lines, Cell Rep. 21 (2017) 1936-1952, https://doi.org/10.1016/j.celrep.2017.10.052.

[30] D.A. Scott, A.D. Richardson, F.V. Filipp, C.A. Knutzen, G.G. Chiang, Z.A. Ronai, A. L. Osterman, J.W. Smith, Comparative metabolic flux profiling of melanoma cell lines: beyond the Warburg effect, J. Biol. Chem. (2011), https://doi.org/10.1074/ jbc.M111.282046.

[31] P.R. Blackburn, Z. Xu, K.E. Tumelty, R.W. Zhao, W.J. Monis, K.G. Harris, J.M. Gass, M.A. Cousin, N.J. Boczek, M.V. Mitkov, M.A. Cappel, C.A. Francomano, J.E. Parisi, E.W. Klee, E. Faqeih, F.S. Alkuraya, M.D. Layne, N.B. McDonnell, P.S. Atwal, Biallelic alterations in AEBP1 Lead to defective collagen assembly and connective tissue structure resulting in a variant of Ehlers-Danlos syndrome, Am. J. Hum. Genet. 102 (2018) 696-705, https://doi.org/10.1016/j.ajhg.2018.02.018.

[32] W. Hu, L. Jin, C.C. Jiang, G.V. Long, R.A. Scolyer, Q. Wu, X.D. Zhang, Y. Mei, M. Wu, AEBP1 upregulation confers acquired resistance to BRAF (V600E) inhibition in melanoma, Cell Death Dis. 4 (2013), https://doi.org/10.1038/ cddis. 2013.441.

[33] M.M.P. Hermans, M.A. Kroos, J. Van Beeumen, B.A. Oostra, A.J.J. Reuser, Human lysosomal $\alpha$-glucosidase: characterization of the catalytic site, J. Biol. Chem. 266 (1991) 13507-13512.

[34] C.A. Maestri, R. Nisihara, H.W. Mendes, J. Jensenius, S. Thiel, I. Messias-Reason, N. S. De Carvalho, MASP-1 and MASP-2 serum levels are associated with worse prognostic in cervical cancer progression, Front. Immunol. 9 (2018), https://doi. org/10.3389/fimmu.2018.02742.
[35] Z. Chen, D. Zhang, F. Yue, M. Zheng, Z. Kovacevic, D.R. Richardson, The iron chelators Dp44mT and DFO inhibit TGF- $\beta$-induced epithelial-mesenchymal transition via up-regulation of N-Myc downstream-regulated gene 1 (NDRG1), J. Biol. Chem. 287 (2012) 17016-17028, https://doi.org/10.1074/jbc. M112.350470.

[36] J.P. Wang, A. Hielscher, Fibronectin: how its aberrant expression in tumors may improve therapeutic targeting, J. Cancer 8 (2017) 674-682, https://doi.org/ 10.7150/jca.16901.

[37] D. Javelaud, V.-I. Alexaki, A. Mauviel, Transforming growth factor- $\beta$ in cutaneous melanoma, Pigment Cell Melanoma Res. 21 (2008) 123-132, https://doi.org/ 10.1111/j.1755-148X.2008.00450.x.

[38] B.I. Ratnikov, D.A. Scott, A.L. Osterman, J.W. Smith, Z.A. Ronai, Metabolic rewiring in melanoma, Oncogene. 36 (2017) 147-157, https://doi.org/10.1038/ onc.2016.198.

[39] G. Cantelli, J.L. Orgaz, I. Rodriguez-Hernandez, P. Karagiannis, O. Maiques, X. Matias-Guiu, F.O. Nestle, R.M. Marti, S.N. Karagiannis, V. Sanz-Moreno, TGF$\beta$-induced transcription sustains amoeboid melanoma migration and dissemination, Curr. Biol. 25 (2015) 2899-2914, https://doi.org/10.1016/j. cub.2015.09.054.

[40] C. Sun, L. Wang, S. Huang, G.J.J.E. Heynen, A. Prahallad, C. Robert, J. Haanen, C. Blank, J. Wesseling, S.M. Willems, D. Zecchin, S. Hobor, P.K. Bajpe, C. Lieftink, C. Mateus, S. Vagner, W. Grernrum, I. Hofland, A. Schlicker, L.F.A. Wessels, R. L. Beijersbergen, A. Bardelli, F. Di Nicolantonio, A.M.M. Eggermont, R. Bernards, Reversible and adaptive resistance to BRAF(V600E) inhibition in melanoma, Nature. 508 (2014) 118-122, https://doi.org/10.1038/nature13121.

[41] L.E. Gentry, M.N. Lioubin, A.F. Purchio, H. Marquardt, Molecular events in the processing of recombinant type 1 pre-pro-transforming growth factor beta to the mature polypeptide, Mol. Cell. Biol. 8 (1988) 4162-4168, https://doi.org/ 10.1128/mcb.8.10.4162.

[42] C.M. Dubois, M.H. Laprise, F. Blanchette, L.E. Gentry, R. Leduc, Processing of transforming growth factor $\beta 1$ precursor by human furin convertase, J. Biol. Chem. 270 (1995) 10618-10624, https://doi.org/10.1074/jbc.270.18.10618.

[43] L. del Amo-Maestro, L. Marino-Puertas, T. Goulas, F.X. Gomis-Rüth, Recombinant production, purification, crystallization, and structure analysis of human transforming growth factor $\beta 2$ in a new conformation, Sci. Rep. 9 (2019), https:// doi.org/10.1038/s41598-019-44943-4.

[44] S. Beck, J.A. Le Good, M. Guzman, N. Ben Haim, K. Roy, F. Beermann, D. B. Constam, Extraembryonic proteases regulate nodal signalling during gastrulation, Nat. Cell Biol. 4 (2002) 981-985, https://doi.org/10.1038/ncb890.

[45] L. Zacchigna, C. Vecchione, A. Notte, M. Cordenonsi, S. Dupont, S. Maretto, G. Cifelli, A. Ferrari, A. Maffei, C. Fabbro, P. Braghetta, G. Marino, G. Selvetella, A. Aretini, C. Colonnese, U. Bettarini, G. Russo, S. Soligo, M. Adorno, P. Bonaldo, D. Volpin, S. Piccolo, G. Lembo, G.M. Bressan, Emilin1 links TGF- $\beta$ maturation to blood pressure homeostasis, Cell. 124 (2006) 929-942, https://doi.org/10.1016/j. cell.2005.12.035.

[46] G. Jenkins, The role of proteases in transforming growth factor- $\beta$ activation, Int. J. Biochem. Cell Biol. 40 (2008) 1068-1078, https://doi.org/10.1016/j. biocel.2007.11.026.

[47] J. Zhu, C.B. Thompson, Metabolic regulation of cell growth and proliferation, Nat. Rev. Mol. Cell Biol. 20 (2019) 436-450, https://doi.org/10.1038/s41580-019 0123-5.

[48] N.N. Pavlova, C.B. Thompson, The emerging hallmarks of Cancer metabolism, Cell Metab. 23 (2016) 27-47, https://doi.org/10.1016/j.cmet.2015.12.006.

[49] I.A. Kovács, K. Luck, K. Spirohn, Y. Wang, C. Pollis, S. Schlabach, W. Bian, D. K. Kim, N. Kishore, T. Hao, M.A. Calderwood, M. Vidal, A.L. Barabási, Networkbased prediction of protein interactions, Nat. Commun. 10 (2019) 1-8, https://doi. org/10.1038/s41467-019-09177-y.

[50] D.S. Pessotti, D. Andrade-Silva, S.M.T. Serrano, A. Zelanis, Heterotypic signaling between dermal fibroblasts and melanoma cells induces phenotypic plasticity and proteome rearrangement in malignant cells, Biochim. Biophys. Acta - Proteins Proteomics. 1868 (2020), https://doi.org/10.1016/j.bbapap.2020.140525.

[51] L.X. Harrington, G.P. Way, J.A. Doherty, C.S. Greene, Functional network community detection can disaggregate and filter multiple underlying pathways in enrichment analyses, in: Pacific Symp. Biocomput, 2018, pp. 157-167, https://doi. org/10.1142/9789813235533_0015. 